BULLETIN Bulletin hispanique

HispaniQuE Université Michel de Montaigne Bordeaux

118-1 | 2016

La Guerre Civile espagnole aujourd'hui (1936-2016)

\title{
Exilio, Historia, Hispanismo
}

Manuel Núñez de Arenas y el Bulletin Hispanique

María José Solanas Bagüés

\section{(2) OpenEdition}

Journals

Edición electrónica

URL: http://journals.openedition.org/bulletinhispanique/4170

DOI: 10.4000/bulletinhispanique.4170

ISSN: 1775-3821

Editor

Presses universitaires de Bordeaux

\section{Edición impresa}

Fecha de publicación: 15 julio 2016

Paginación: 15-30

ISBN: 979-10-300-0058-0

ISSN: 0007-4640

Referencia electrónica

María José Solanas Bagüés, « Exilio, Historia, Hispanismo », Bulletin hispanique [En línea], 118-1 | 2016,

Publicado el 15 julio 2019, consultado el 08 septiembre 2019. URL : http://journals.openedition.org/

bulletinhispanique/4170; DOI : 10.4000/bulletinhispanique.4170 


\title{
Exilio, Historia, Hispanismo: Manuel Núñez de Arenas y el Bulletin Hispanique
}

\author{
María José Solanas BAgüÉs \\ Universidad de Zaragoza - España
}

Manuel Núñez de Arenas (1886-1951), intellectuel espagnol et historien, a entretenu, lors de ses exils à Bordeaux, des liens universitaires étroits avec le cercle des hispanistes dirigé par Georges Cirot autour du Bulletin Hispanique. Cette analyse met en relation le développement de l'histoire de l'historiographie espagnole réalisée en exil avec son rôle d'intermédiaire culturel.

Mots-clés: Núñez de Arenas, exil, historiographie, intermédiaire culturel.

Manuel Núñez de Arenas (1886-1951) fue un historiador e intelectual español que mantuvo durante sus exilios en Burdeos una estrecha conexión académica con el círculo de hispanistas encabezado por Georges Cirot en torno al Bulletin Hispanique. Este análisis conecta el desarrollo de la historia de la historiografía española realizada en el exilio con su papel como intermediario cultural.

Palabras claves: Núñez de Arenas, exilio, historiografía, intermediario cultural.

Manuel Núñez de Arenas (1886-1951), Spanish intellectual and historian, was, during his exile times in Bordeaux, in constant academic contact with the Hispanists circle run by Georges Cirot, round the Bulletin Hispanique. The present analysis connects the development of the Spanish historiography history, achieved in exile, to its role of cultural intermediary.

Keywords: Núñez de Arenas, Spanish republican exile, historiography, cultural mediator.

$\mathrm{M}$ anuel Núñez de Arenas falleció en el exilio, en París, el 9 de septiembre de 1951. Una necrológica en la revista Bulletin Hispanique evidenciaba su estrecha relación con la historiografía francesa, los hispanistas galos y la 
Facultad de Letras de Burdeos ${ }^{1}$. A lo largo de estas páginas analizaremos la trayectoria intelectual de Núnez de Arenas, incidiendo en las etapas en las que desarrolló su labor como historiador: sus exilios de 1923 y 1939 en Francia. Si bien en su caso el destierro habilitó el escenario idóneo para convertirse definitivamente en un historiador, también fue objeto de la represión directa internacional ejercida en la década de los años cuarenta del siglo XX sobre los historiadores, tal y como veremos en el episodio de su denuncia y posterior detención por la Gestapo ${ }^{2}$.

De igual manera, el estudio de las redes establecidas entre las diferentes coordenadas historiográficas que transitó, nos permitirá situarlo en el proceso de formación de la historiografía española del siglo XX, conectándolo con las aportaciones y el desarrollo de la profesión realizadas fuera de las fronteras nacionales. La figura y la obra de Manuel Núnez de Arenas fueron olvidadas debido a la ruptura histórica que supuso «la hora cero» de 1939. Sólo a partir de los años 60 volvieron a ser reivindicadas por sus nuevos discípulos en suelo francés: jóvenes hispanistas como Robert Marrast, o exiliados españoles como Manuel Tunón de Lara, quienes reintroducirán sus textos en España, completando una transferencia historiográfica iniciada a principios del siglo $\mathrm{XX}^{3}$.

\section{Apuntes biográficos de Manuel NúÑez de Arenas:} FORMACIÓN Y COMPROMISO

Cuando Manuel Núñez de Arenas se refugió en Burdeos en 1923 con 37 años no se definía como historiador, ni era percibido como tal en la comunidad profesional gestada en Espańa en este primer cuarto de siglo ${ }^{4}$. Pese a que ya había escrito el estudio sobre el movimiento obrero que lo convertiría en un precursor décadas después, la cantidad de actividades reseńables que desarrolló en este primer cuarto de siglo -militante político, periodista, impulsor de la Escuela Nueva, traductor- no lo situaban en la órbita de la profesión histórica, sino que transitaba itinerarios propios de lo que podría definirse como un «intelectual comprometido». Esta situación cambió al cruzar la frontera hacia

1. Charles V. Aubrun, «Manuel Núńez de Arenas y de la Escosura (1886-1951)», Bulletin Hispanique, 53-4, 1951, p. 459-461. Le precede la necrológica de uno de sus historiadores de referencia, Rafael Altamira, fallecido en el exilio mejicano, y que en un principio iba a redactar el propio Núńez de Arenas. Marcel Bataillon, «Rafael Altamira y Crevea (1866-1951)», Bulletin Hispanique, 53-4, 1951, p. 457-459. Una primera versión de este texto fue presentado para su discusión en el XI Congreso de la Asociación de Historia Contemporánea celebrado en la Universidad de Granada en septiembre de 2012.

2. Antoon de Baets, «Exile and Acculturation: Refugee Historians since the Second World War», The International History Review, XXVIII, 2, june 2006, pp. 316-349.

3. El artículo fundacional sobre la perspectiva de las transferencias culturales en Michel Espagne y Michel Werner, «La construction d'une référence culturelle allemande en France. Genèse et histoire (1750-1914)», Annales ESC, 4, juillet-août 1987, p. 969-992.

4. Ignacio Peiró Martín, Historiadores en España. Historia de la historia y memoria de la profesión, Zaragoza, Prensas de la Universidad de Zaragoza, 2013. 
Francia. Pero antes de llegar a ese punto, es conveniente diseccionar brevemente un relato de vida, hasta ahora evocado más que investigado5.

Manuel Núñez de Arenas y de la Escosura había nacido en Madrid el 1 de abril de 1886. Entre sus inquietos antepasados familiares - conocemos las trayectorias de los varones, muy poco las de ellas-, podemos encontrar antecedentes profesionales que él mismo reproducirá en sus múltiples intereses intelectuales. Toda una pléyade de figuras decimonónicas -su bisabuelo Espronceda, su tío abuelo Patricio de la Escosura, José Núñez de Arenas o Cristino Martos entre otros- que compaginaban ideas liberales, cargos políticos, exilios, vocaciones históricas, proyectos periodísticos, cátedras universitarias y sillones académicos con veleidades literarias más o menos destacadas.

A diferencia de su familia, Manuel Núñez añadió un sesgo que condicionó su evolución intelectual y profesional. Tempranamente se decantó por ingresar en las filas del movimiento obrero, y se interesó especialmente por las manifestaciones culturales y pedagógicas concebidas de manera heterogénea y paralela a los espacios institucionales oficiales: sociedades, casas del pueblo, escuelas societarias y canales alternativos para la difusión de una nueva cultura obrera.

Fue su pertenencia a una familia burguesa y cosmopolita lo que le permitió una elitista educación francófona. Tras cursar el bachillerato en los Jesuitas de Chamartín de Madrid, en 1901 obtuvo el diploma de «Lengua y civilización francesa» en el Lycée Montaigne de la Universidad de Burdeos ${ }^{6}$. Durante sus inacabados estudios universitarios en Suiza mantuvo el primer contacto con las organizaciones obreras y con la figura de Georges Renard. Un modelo de intelectual comprometido a cuya obra Syndicats, Trade-Unions et Corporations (1909) añadió en su traducción de 1916 las «Notas sobre el movimiento obrero español», convirtiéndolo en una referencia transgeneracional. En el prólogo, nuestro autor rememoraba su estancia en Lausana, y el origen simbólico de los dos vectores que perfilarían sus actuaciones, la cultura obrera y la universitaria:

[...] de estudios tan diferentes de los actuales, de iniciación en doctrinas políticas, de contacto con el proletariado, de conocimiento de la organización obrera.

5. Informaciones que aportaron los autores que recuperaron su obra, el primero de ellos Robert Marrast en sus Prólogos a Manuel Núñez de Arenas, L'Espagne des Lumières au Romantisme, Paris, Centre de Recherches de l'Institut d'Études Hispaniques, 1963; Manuel Núnéz de Arenas y Manuel Tunón de Lara, Historia del movimiento obrero español, Barcelona, Nova Terra, 1970. También en M. Tuñón de Lara, Medio siglo de cultura española, 1885-1936, Madrid, Tecnos, 1970; Javier Malagón, «Los historiadores y la historia en el exilio», en José Luis Abellán, El exilio español de 1939, Madrid, Taurus, 1978, vol. V, p. 245-353, en p. 311-312, y más recientemente Ignacio Peiró y Gonzalo Pasamar, Diccionario Akal de Historiadores Españoles Contemporáneos (1840-1980), Madrid, Akal, 2002, p.446-447, o Alicia Alted, «Historiografía e historiadores en el exilio", en Ma Fernanda Mancebo (ed.), Encuentros de Historia y Literatura. Max Aub y Manuel Tuñón de Lara, Valencia, Biblioteca Valenciana, Generalitat Valenciana, Conselleria de Cultura i Educació, Direcció General del Llibre, Arxius i Biblioteques, 2003.

6. Manuel Tuñón de Lara, Medio siglo de cultura española, op. cit., p. 163. 
Era yo casi un niño, seguía la carrera de ingeniero, y robaba horas á las matemáticas para asistir á la Casa del Pueblo y á la Universidad. Y en aquella Casa del Pueblo y en aquella Universidad oí el nombre de Georges Renard ${ }^{7}$.

La figura de Renard se erigía para él en paradigma del intelectual socialista, que desde la segunda mitad del siglo XIX aunó investigación y práctica en su interés por las teorías sociales y la literatura. Además de la publicación de sus obras, lo hizo desde sus clases en la Universidad de Lausana y el Collège de France sobre "Histoire du Travail», la creación de la Revue Socialiste, o como fundador de la Casa del Pueblo. Y de hecho, si bien el español nunca alcanzó las mismas cotas de reconocimiento académico, podemos identificar puntualmente itinerarios semejantes en su trayectoria vital e intelectual.

A partir de su regreso a España y hasta 1923, Núñez de Arenas dividirá sus esfuerzos entre varios campos de actuación. Por un lado, su formación e inserción en el mundo universitario: la licenciatura en Filosofía y la lectura de la tesis, los intentos por acceder a la docencia mediante oposiciones, así como por conseguir una pensión de la JAE. Por otro lado, su cada vez más intensa actividad política, mediante experiencias como la Escuela Nueva y la Escuela Societaria, colaboraciones y creación de publicaciones, o la militancia activa en el PSOE y con el sector escindido que funda el PCOE. Ambos itinerarios coexistirán, con alternancia de picos de actuación en el campo académico y en el político-cultural, aunque quizás más acentuado o con más visibilidad este segundo escenario de las ideologías prácticas en el convulso Madrid de la segunda etapa de la Restauración.

Núñez de Arenas se licenció en Filosofía el 4 de septiembre de 1911 en la Universidad de Madrid. Cuatro años después, el 16 de junio de 1915, defendió su tesis, con la que obtuvo Sobresaliente y Premio Extraordinario: «Un reformador social. Ramón Dionisio de la Sagra y Perig». De manera consecuente con sus intereses intelectuales, la elección del tema respondía al intento por reflejar a través de su biografiado el siglo XIX europeo, «la historia de múltiples disciplinas científicas» $\mathrm{y}$ «de las ideas sociales en España» ${ }^{8}$, el deseo de valorar justamente la intermediación cultural, y la intención de poner en valor el papel de los propios mediadores. En este sentido, en la «Necrológica» de su maestro Georges Cirot en 1947 recordaba:

Ya sé que estos hombres con sus aportaciones enriquecen su patria, pero al tiempo enriquecen la ajena, dándole un área de mayor influencia. Debían pues, ser mirados a la vez en los dos países como hijos preclaros.

7. Manuel Núnez de Arenas, «Prólogo» a Georges Renard, Sindicatos, Trade-Unions y Corporaciones, Madrid, Daniel Jorro Editor, 1916, p. V.

8. Prólogo a la publicación de su tesis «Don Ramón de la Sagra, reformador social», Revue Hispanique, París, LX, 1924, p. 329-531, las citas en la p. 330. Jean-Louis Guereña considera a Núnez de Arenas el primero en cultivar la historia social en España con esta investigación, en "Entre Francia y Espańa. Vivencias y reflexiones sobre un itinerario», Roberto García Cárcel y Eliseo Serrano Martín (eds.), Exilio, memoria personal y memoria histórica. El hispanismo francés de raiz española en el siglo XX, Zaragoza, IFC, 2009, p. 177-201. 
Pero la idea es difícil de aceptar. Siempre me acordaré de una pequeña discusión que se originó al examinar mi tesis doctoral. Trataba yo de Sagra, gran transportador de ideas a España y decíame Ortega y Gasset, que hombre sin sistema personal, original, no merecía estudio. Inmediatamente acudió en defensa de mi punto de vista contrario Cossío mostrando cuánto esta corriente de ideas marcaba en una civilización. [...]

Muchas veces he pensado en la injustificada preterición con que se considera en todos los países a tres categorías de hombres intelectuales que, sin embargo, contribuyen tremendamente a la civilización [...], a los xenófilos, a los traductores y a los divulgadores de teorías extranjeras.

Sólo cuando pasan varios siglos se atreven los historiadores a reconocer el mérito de esos hombres laboriosos, modestos y entusiastas ${ }^{9}$.

En este sentido, él mismo contribuyó como traductor ocasional a la circulación internacional de la literatura. Los textos traducidos parecen obedecer tanto a una afinidad intelectual con los contenidos, un interés en la difusión de ideas y teorías sociales y filosóficas, como a un medio para ganarse la vida, todas ellas razones aplicables a otros intelectuales e historiadores de principios de siglo que se dedicaron a la traducción ${ }^{10}$.

También durante estos años intentó sin éxito acceder al «mercado universitario europeo» ${ }^{11}$ mediante una de las pensiones de la nueva institución que permitía a licenciados y profesores ampliar estudios en el extranjero: la Junta para la Ampliación de Estudios ${ }^{12}$. Optó a las ayudas desde la primera convocatoria en 1907 (para estudiar la cultura y literatura francesas en Francia, Bruselas y Ginebra), e insistió en 1910 («La enseñanza y el estado de los estudios filosóficos» en las Universidades de Montpellier, Nancy y París), y 1913 (suponemos que algo desencantado, escribe unas escuetas líneas solicitando pensión para «ampliar sus conocimientos»). La conseguirá finalmente en 1928, cuando ya residía fuera de España, así como en 1935, en peticiones donde ya define su condición de historiador.

Para finalizar este apretado recorrido por su etapa de formación citaremos sus intentos por integrar el mundo de la docencia universitaria y media: las

9. Manuel Núñez de Arenas, «Un gran hispanista desaparece. Monsieur le Doyen Georges Cirot», Boletín de la Unión de Intelectuales Españoles, París, 33-34-35, agosto-septiembre-octubre 1947, p. 3-4.

10. Las obras traducidas son: Émile Hennequin, La critica cientifica, Biblioteca Científica de Filosofía, Daniel Jorro, 1909; Adolfo Prins, Criminalidad y represión: ensayo de ciencia penal, Madrid, Hijos de Reus, 1911; Alfred Weber, Historia de la Filosofia europea (séptima edición), Madrid, Daniel Jorro, 1914; Gabriel Dromard, El Ensueño y la acción, Madrid, Librería Gutemberg de José Ruiz, 1915, la de G. Renard en 1916 y Egdar Quinet, Mis vacaciones en España, Madrid, Ediciones La Nave, 1931.

11. Esta idea y su desarrollo en Victor Karady, «La République des lettres des temps modernes. L'internationalisation des marchés universitaires occidentaux avant la Grand Guerre», Actes de la Recherche en Sciences Sociales, 121-122, Mars 1998, p. 92-103.

12. De la abundante bibliografía sobre la JAE impulsada sobre todo desde su centenario, citaremos solamente Miguel Ángel Puig-Samper (coord.), Tiempos de investigación: JAE-CSIC, cien años de ciencia en España, Madrid, CSIC, 2007. 
oposiciones. Los sucesivos fracasos no hicieron mella en su empeño ni parecen haberle llevado al rechazo total del sistema docente.

Asistiendo en calidad de candidato a la plaza de las enconadas oposiciones a la cátedra de Sociología de la Central en 1916 -ganadas por Severino Aznar frente al otro aspirante José Castillejo-, pudo imaginar las escasas posibilidades de entrar en la Universidad para un miembro reconocido del PSOE ${ }^{13}$. Pese a sus ilustres antepasados universitarios y las amistades con catedráticos universitarios, las relaciones de poder quedaban muy lejos para un recién doctorado, traductor y articulista, pero sobre todo conocido por su activa labor dentro del movimiento obrero y como promotor, bibliotecario o Presidente de la Escuela Nueva de la Casa del Pueblo ${ }^{14}$.

No en vano, las relaciones y redes de contactos establecidas por Manuel Núñez de Arenas en este primer cuarto de siglo no estaban dirigidas a la inserción en la comunidad de historiadores que estaba gestándose en Espańa -y de manera más sobresaliente en Madrid-, sino que respondían a inquietudes propias de un intelectual publicista, militante activo del $\mathrm{PSOE}^{15}$, que intentó desde sus centros de formación crear un nexo estable entre obreros e intelectuales. Sus contactos con miembros del Centro de Estudios Históricos o de la Universidad, los nuevos focos pautadores del oficio del historiador, se realizaron al margen de estas instituciones, en el marco de las conferencias del Ateneo de Madrid, y de las iniciativas de la Escuela Nueva ${ }^{16}$.

Sus planteamientos sobre educación, política y sociedad pueden rastrearse en sus colaboraciones para los periódicos y revistas en los que colaboraba: escribió en el El Socialista -órgano oficial del PSOE-, en el semanario España durante la época en que fue dirigido por Luis Araquistáin, participó en la redacción de

13. Sobre la polémica que generaron estas oposiciones, vid. Ignacio Peiró, "Los aragoneses en el Centro de Estudios Históricos: historia de una amistad, historia de una escuela, historia de una profesión", en José-Carlos Mainer (ed.), El Centro de Estudios Históricos (1910) y sus vinculaciones aragonesas (con un homenaje a Rafael Lapesa), Zaragoza, IFC - Sociedad Estatal de Conmemoraciones Culturales, 2010, p. 135-171, en p. 150 y ss.

14. Francisco de Luis, «Lecturas y lectores en la Casa del Pueblo de Madrid», CEE Participación Educativa, número extraordinario (2010), p. 67-90.

15. Ingresó en la Agrupación Socialista (AS) de Madrid el 1 de enero de 1911. Representó a la AS de Toledo y a la Sociedad de Agricultores de Mora (Toledo) en el IX Congreso del PSOE en 1912; a la AS de Minas de Riotinto (Huelva) en el X Congreso en 1915; a la AS de Madrid y a la Escuela Nueva en el XI Congreso en 1918 y a la Escuela Nueva en el Congreso Extraordinario en 1919. Perteneció a la Comisión Ejecutiva del PSOE de 1918 a 1921, siendo secretario de estudios en 1918-1919 y vocal de 1919 a 1921. Asistió al XIV Congreso de la UGT en 1920 como delegado del Sindicato de Profesiones Liberales de Madrid. Partidario de la Tercera Internacional fue dado de baja en el PSOE en mayo de 1921. Fue miembro del Comité Central del PCE y candidato por Madrid de este Partido en las elecciones generales de 1923. Datos extraídos de la voz «Núñez de Arenas y de la Escosura, Manuel» Diccionario Biográfico de la Fundación Pablo Iglesias, [en línea]. Fundación Pablo Iglesias [Página consultada el 1 de abril de 2014]. Acceso en: http://www.fpabloiglesias.es/archivo-y-biblioteca/diccionario-biografico/ biografias/13735_nunez-arenas-y-escosura-manuel

16. Jean-Luis Guereña, "Cultura y política en los años diez: Ortega y La Escuela Nueva», Cuadernos Hispanoamericanos, 403-405, enero-marzo 1984, p. 544-563. 
La Antorcha, y en la creación, secretaría y dirección del semanario comunista La Internacional (1919-21).

Fue mediante la aplicación práctica de esta concepción «horizontal» de la transmisión de la cultura como Manuel Núnez de Arenas ensayó la consolidación de un centro mixto en el que difundir teorías y realidades, con el objetivo de introducir la actividad intelectual entre las bases socialistas. El proyecto, materializado en la Escuela Nueva, contó con la implicación de varios historiadores procedentes de la Universidad, tanto en su creación como en las diferentes actividades y ciclos sobre "historia socialista».

De la intersección de estos dos itinerarios surge en 1916 «Notas sobre el movimiento obrero español», el apéndice a la obra de G. Renard. Con estos antecedentes académicos, no debe extrañar que su elaboración superase las anteriores historias de partido y utilizase recursos de la historiografía profesional, convirtiéndose según David Ruiz en el «primer estudio histórico del movimiento obrero desde sus inicios hasta la Primera Guerra Mundial con inequívocas pretensiones de cientificidad ${ }^{17}$. Pocos contemporáneos dudarían de la idoneidad de Núñez de Arenas para llevar a cabo la labor de guardián de la historia del movimiento obrero. Sin embargo, el exilio finalizó con este proyecto, abriendo la puerta a una reinvención académica y profesional en suelo francés.

\section{EN LA SENDA DE LA HISTORIA: PRIMER EXILIO EN BURDEOS}

El primer exilio de Manuel Núñez de Arenas debe ser analizado como un inicial refugio en Burdeos para evitar la condena a ocho ańos de cárcel tras la publicación de un artículo contrario a la guerra de Marruecos, que devendrá en exilio al iniciarse la dictadura de Primo de Rivera, y se convertirá en una «estancia» gracias a una pensión de la JAE en 1928.

En enero de 1923 ya se ha instalado en Burdeos. La condena que pesa sobre él no es la primera, ya había sido encarcelado durante la huelga de 1917 y de nuevo en $1922^{18}$. Así mismo, era un destacado "tercerista», miembro fundador y candidato del Partido Comunista, un significado intelectual que podía encontrar problemas, siendo más que probable una nueva detención. Como hicieran sus antepasados liberales en el siglo anterior, tomaba el incierto camino del exilio, en su caso a la ya conocida ciudad de Burdeos.

Durante este primer destierro Núnez de Arenas, en palabras de Tunón de Lara, «intensificó su relación con la historia» ${ }^{19}$. Es más, su propia elaboración

17. «De Núñez de Arenas al pluralismo metodológico. Todo en menos de un siglo», Millars. Espai $i$ historia, 27, 2004, pp. 89-100, la cita en p. 90; Jorge Uría, «La historia social y el contemporaneísmo espańol. Las deudas del pasado», Revista de Historia Jerónimo Zurita, 71, 1997, p. 95-141; Francisco Sánchez, «El movimiento obrero antes de la guerra civil: el enfoque de Manuel Tuñón de Lara», Cuadernos de Historia Contemporánea, 30, 2008, p. 105-116.

18. Detenido en Bilbao tras la huelga general de agosto de 1917 con Luis García Bilbao, y conducidos al buque "Alfonso XIII». Su detención tras el atentado a Dato y su pronta liberación en 1922 en El Globo, (Madrid), 23 de noviembre de 1922, p. 1.

19. Manuel Tuñón de Lara, Medio siglo de cultura española, op. cit., p. 164. 
de la imagen de historiador reforzó el conjunto de estrategias encaminadas a lograr la identidad profesional que desplegó durante estos años. En su caso, el exilio lo situó en un escenario más idóneo que le proporcionó las herramientas necesarias para profundizar en la profesionalización de la historia a través de la deseada carrera docente y la consecución de una pensión de la JAE, la investigación en los archivos y bibliotecas, el establecimiento de contactos con otros historiadores, y la publicación en revistas especializadas a ambos lados de la frontera.

La redefinición de su vida alcanzó también la esfera personal, pues contrajo matrimonio con Geneviève Elisabeth Lafitte en 1927. Y de igual manera, pudo participar del clima intelectual de la ciudad de Burdeos primero y de París después, relacionándose con otros asilados europeos que se incorporaban a las secciones de estudios extranjeros que las facultades y centros de enseñanza ofrecían a las diferentes colonias de refugiados ${ }^{20}$.

Burdeos suponía en 1923 uno de los centros más activos del hispanismo francés, todavía bajo la orientación de Alfred Morel-Fatio y Mérimée. Para comprender el desarrollo del hispanismo en torno a la Facultad de Letras de la Universidad de Burdeos citaremos cuatro hitos representativos: en 1898 se había creado la "agrégation» de español, en 1899 apareció el Bulletin Hispanique, en 1908 se creó el Instituto francés de Madrid y en 1928 la Casa de Velázquez ${ }^{21}$.

Para Núnez de Arenas suponía el retorno a la ciudad donde cursó estudios con uno de sus maestros, Georges Cirot. De inmediato se puso en contacto con dicho hispanista, discípulo de Alfred Morel-Fatio, doctorado con una tesis sobre el Padre Mariana, y convertido en el primer catedrático de Langue et littérature espagnole de la Universidad de Burdeos (1898). Animado por él, comenzó en octubre de 1923 una breve relación epistolar con A. Morel-Fatio; breve debido a la enfermedad y fallecimiento en 1924 del conocido como "padre» del hispanismo científico francés. Para un historiador no profesional como Núnez de Arenas debió de resultar cuando menos estimulante el contacto iniciado con el renombrado especialista francés -y tan opuesto ideológicamente-, a quien pidió consejos e hizo partícipe de sus investigaciones y proyectos sobre el objeto de estudio común a ambos: españoles refugiados en Francia desde la Revolución Francesa y durante todo el siglo XIX. De hecho, lejos de la actividad política y periodística que llevaba a cabo en España, su inmersión en la tarea de «rétablir

20. J. Bechstein, "L'œuvre de Kafka à Bordeaux, ou la vie de Jean et Charlotte Carrive à la Gironde», A. Ruiz (dir.), Présence de l'Allemagne à Bordeaux du siècle de Montaigne à la veille de la Seconde guerre mondiale. Hommage au Goethe-Institut de Bordeaux à l'occasion de son $25^{\mathrm{e}}$ anniversaire. Bordeaux, Presses Universitaires de Bordeaux, 1997, pp. 221-231, quien señala la amistad de la pareja -traductores de la obra de Kafka al francés, y ella profesora en un instituto de Burdeos- con el historiador Manuel Núnez de Arenas en el periodo de entreguerras.

21. Citaremos los monográficos de las revistas Ayer, 31, 1998; Historia Contemporánea, 20, 2000; Arbor, CLX-VIII, 64, 2001, y las obras de conjunto: B. Pellistrandi (ed.), La historiografía francesa del siglo XX y su acogida en España, Madrid, Casa de Velázquez, 2002; Francisco García (ed.), La historia moderna de España y el hispanismo francés, Madrid, Marcial Pons-Facultad de Humanidades de Albacete UCLM, 2009; Joaquín Álvarez Barrientos (ed.), Memoria del hispanismo. Miradas sobre la cultura española, Madrid, Siglo XXI, 2011. 
la vérité à coups de documents» ocupaba ahora todo su tiempo: «[...] je suis tellement obsédé par mes recherches que je ne parle que de Ferdinand VII et je ne jure que par mes réfugiés ${ }^{22}$.

No fue la única puerta que le abrió Georges Cirot. El órgano difusor del hispanismo del sur de Francia, el Bulletin Hispanique, fue creado en 1899 por Alfred Morel-Fatio (1850-1924), Ernest Mérimée (1846-1924), Pierre Paris (1859-1931), Georges Radet (1859-1941) y Georges Cirot (18701946), tras la unión de Annales de la Faculté de Bordeaux (1879) y la Revue des Universités du Midi (1895). Esta publicación trimestral sirvió de tribuna tanto para especialistas franceses en Italia, España, Portugal o Latinoamérica, como para los propios historiadores y filólogos de los territorios investigados. Entre 1923 y 1930, 45 autores firmaron los 92 artículos de la sección principal en los 24 números aparecidos. Seis de los autores eran españoles (1 de ellos, Miguel Romera Navarro, desde su puesto en la Universidad de Pennsylvania): Américo Castro (uno en 1923); Carmelo Viñas Mey (un largo artículo sobre los afrancesados que se publicó a lo largo de 3 números entre 1923 y 1924); Ramón Menéndez Pidal (uno en 1925); Justo Pérez de Urbel (un artículo a lo largo de 4 números en 1926, y otro en 1930); y Claudio Sánchez Albornoz (uno en el último número de 1930).

Sin embargo, la autoría de Núñez de Arenas está presente en otras 2 secciones de la revista: «Variétés» $\mathrm{y}$ «Bibliographie» (Gráficos 1 y 2). En la primera tenían cabida artículos más breves que los principales, y la segunda contenía las reseñas, un espacio donde se alternaban firmas de los autores consagrados «de la casa»" ${ }^{23}$ con las de los alumnos que se iniciaban en el aprendizaje de la publicación en medios especializados.

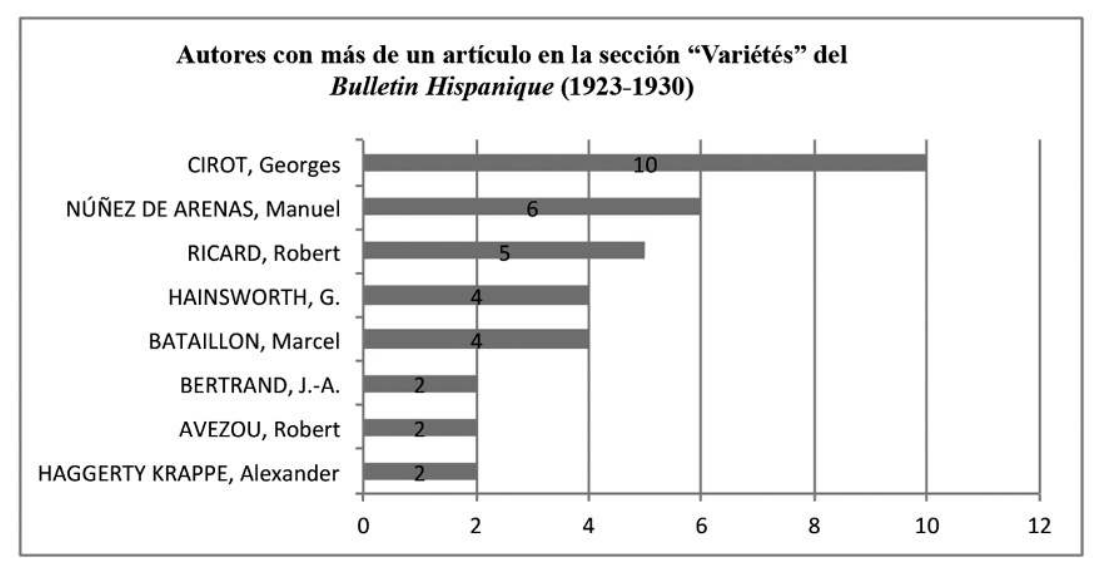

Gráfico 1 : Autores con más de un artículo en la sección "Variétés” el Bulletin Hispanique. Fuente: Elaboración propia a través de la revista.

22. Carta de Manuel Núñez de Arenas a Alfred Morel-Fatio, 28-10-1923, en la Bibliothèque de Versailles (Fondo AMF). Agradezco al profesor Igancio Peiró el haberme permitido su consulta, y remito a la próxima publicación de su Epistolario.

23. No en vano, Georges Cirot, secretario de la publicación es el autor de 117 reseńas de las 265 publicadas durante este periodo. 


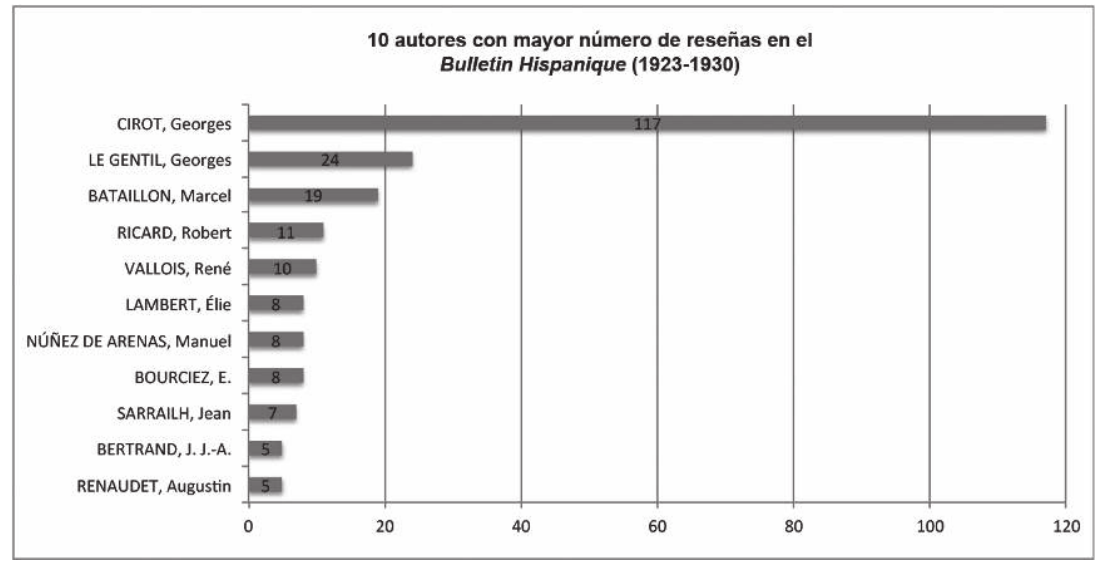

Gráfico 2 : Los 10 autores con mayor número de reseñas en el Bulletin Hispanique. Fuente: Elaboración propia a través de la revista.

Unas colaboraciones -más distanciadas a partir de 1928, cuando se trasladó a París pensionado por la JAE-, que no le impidieron publicar también en la "rival" Revue Hispanique de Foulché-Delbosch, comenzando por su tesis doctoral en $1924^{24}$. De igual manera, su estrategia para alcanzar el reconocimiento como historiador en Espańa le llevó a ver impresos sus primeros artículos en publicaciones especializadas: la Revista de Filología Española dirigida por Ramón Menéndez Pidal25, el Boletín de la Biblioteca Menéndez y Pelayo o la Revista de la Biblioteca, Archivo y Museo del Ayuntamiento de Madrid.

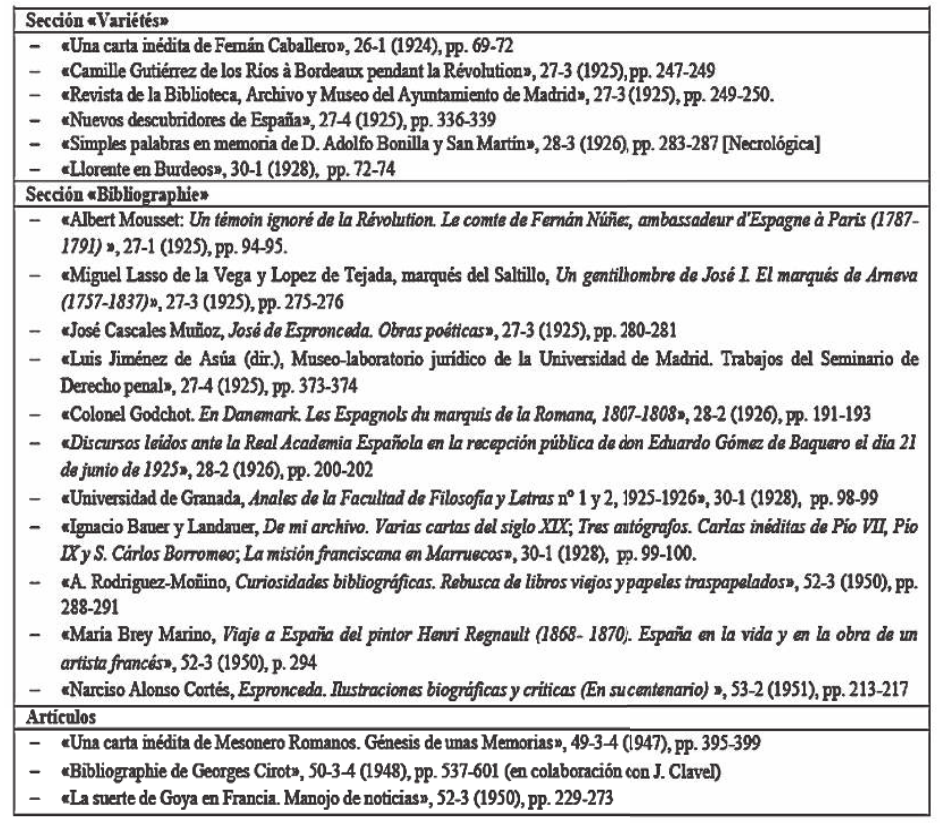

Tabla 1 : Publicaciones de M. Núñez de Arenas en el Bulletin Hispanique

24. A. Niño, Cultura y diplomacia. Los hispanistas franceses y España 1875-1931, Madrid, CSIC, 1988. Además de su tesis en 1924, publicó en 1925, 1929, y en el último número dedicado a la memoria de su fundador, en 1933.

25. El propio Menéndez Pidal recomendó en 1929 la prórroga de su pensión. 
Emplazando para otro lugar un análisis más exhaustivo de sus publicaciones, redes establecidas y estrategias de inserción y reconocimiento ${ }^{26}$, solamente enunciaremos aquí sus investigaciones sobre los afrancesados, su interés por rehabilitar el siglo XIX español de manera rigurosa y su contribución a la «historia nacional» encaminada hacia «la aportación intelectual de Espańa a Francia en el primer tercio del siglo XIX».

Rastreando los fondos documentales consultados podemos reconstruir la red de archivos y bibliotecas visitados, de eruditos e historiadores locales del Sur de Francia, estudiosos y conservadores orgullosos de un pasado nacional al que también contribuyeron los departamentos alejados de París. La concepción de la investigación que Núñez de Arenas proponía para el estudio del XIX español así lo requería:

Nuestra historia del siglo XIX se ha escrito hasta ahora, en general, sea por actores de los sucesos, sea por literatos que han despreciado la rebusca paciente en los archivos. Además, nuestra perpetua guerra civil hace imprescindible el bucear, no sólo en los archivos espańoles, sino en muchos del extranjero. De un laborioso acopio de documentos podrá salir un día la verdadera narración de los sucesos del siglo pasado ${ }^{27}$.

Cumpliendo Núñez 40 años en 1926, la estrategia de inserción en el marco hispanista bordelés cambió de rumbo al instalarse él en París, y desde allí iniciar una serie de acciones que le conducirán de nuevo a Espańa. Varios fueron los episodios que permiten rastrear dicha evolución. Sus contactos epistolares con la Real Academia de la Historia habían dado fruto, y en 1926 apareció su «Informe sobre la expropiación de tumbas españolas en Burdeos» requerido por la $R A H$ tras sus gestiones ${ }^{28}$. Como consecuencia de este ejercicio, Núñez de Arenas se convirtió en orgulloso correspondiente de la Academia. Al año siguiente, su tesis sobre Ramón de la Sagra fue declarada obra de utilidad pública por la Real Academia de Ciencias Morales y Politicas ${ }^{29}$.

Si bien se había mantenido un atisbo de presencia en la vida cultural madrileña a través de sus artículos sobre personajes románticos en el exitoso rotativo vespertino $L a V o z$, así como varias colaboraciones en revistas minoritarias como Alfar o Verba, en 1928 se intensificó. Retornó a España para realizar unas oposiciones de Catedrático de "Lengua francesa», coincidiendo con la petición de una pensión a la JAE que por fin obtendría. Tras pedir la excedencia de su recién conseguida cátedra de Instituto, dedicará dos años a la investigación en archivos y bibliotecas de Francia.

26. Tema desarrollado en mi Tesis Doctoral (Universidad de Zaragoza), donde amplio la bibliografía aportada en este texto.

27. Manuel Núñez de Arenas, «Españoles fuera de España. La expedición de Vera en 1830», Boletín de la Real Academia de la Historia, Madrid, 1926, p. 243-291, la cita en p. 244 de la reproducción en 1963.

28. Boletín de la Real Academia de la Historia, Madrid, 1926, p. 314-329.

29. El 6 de diciembre de 1927, publicado en la Gaceta de Madrid, no 342, 08/12/1927, p. $1467-1468$. 
Sorprendentemente, tras haber alcanzado casi todos sus objetivos, durante los años de la República y de la guerra civil asistimos a un retraimiento de su labor como historiador. Es tiempo de dedicarse a la enseńanza en los Institutos Velázquez de Madrid y de Alicante. Además de su confesada vocación didáctica, también es posible que sufriera un cierto desencanto al no lograr la deseada inserción en la comunidad de historiadores de la península, y no verse reconocido como un igual salvo por contadas excepciones. Durante la guerra civil, ejerció como inspector de enseñanza, y participó en la experiencia de los frustrados Institutos Obreros y la Casa de Cultura de Valencia hasta su nueva salida del país, esta vez definitiva.

\section{GuERRA, PRISIÓN Y EXILIO DEFINITIVO (1939-1951)}

Como sucedió en otras áreas científicas, la dictadura cercenó el desarrollo de la historiografía profesional conformada en torno a la investigación y la docencia universitaria, produciéndose lo que Ignacio Peiró y Miquel Marín han denominado la "hora cero» de la historiografía, comenzando la «larga travesía en el desierto» ${ }^{30}$. En cuanto a Núñez de Arenas, sufrió la máxima sanción impuesta por las comisiones de depuración de los profesores de institutos de segunda enseńanza, la separación del servicio ${ }^{31}$. Para entonces ya había tomado el camino del segundo e irreversible exilio ${ }^{32}$, donde retomará su labor de historiador, no sin antes sufrir el episodio que detallamos a continuación.

En el caso del profesor madrileño, la red de relaciones tejida en sus anteriores estancias en la Universidad de Burdeos le facilitó el acceso a una plaza de Lector de español. Allí se reencontró con los hispanistas y maestros que tuvo durante los años 20, y que habían convertido el modelo de hispanista "vigilante» en una suerte de "hispanismo cordial» representado por Georges Cirot, M. Bataillon o Gaspard Delpy, que en su máxima expresión se dedicaron a prestar ayuda a los refugiados (además del protagonista de nuestro artículo, la Universidad de Burdeos acogió a Claudio Sánchez Albornoz y Ramón Menendez Pidal).

30. Ignacio Peiró, «Historiadores en el purgatorio. Continuidades y rupturas en los años sesenta», Cercles. Revista d'Història Cultural, Universitat de Barcelona, 16, 2013, pp. 53-81; Miquel À. Marín Gelabert, «Revisionismo de Estado y primera hora cero en España, 19361943", Carlos Forcadell, Ignacio Peiró y Mercedes Yusta (eds.), El pasado en construcción. Revisionismos históricos en la historiografía contemporánea, Zaragoza, IFC, 2015, pp. 363-406.

31. «Orden de 29 de julio de 1939, separando definitivamente del servicio a los catedráticos de Universidad e Instituto que se mencionan", BOE (18/08/1939), p. 4542. Olegario Negrín, «La depuración del profesorado de los institutos de segunda enseñanza. Relación de los expedientes resueltos por el Ministerio de Educación Nacional (1937-1943)", Historia de la educación. Revista interuniversitaria, 24, 2005, pp. 503-542.

32. De la abundante biliografía aparecida en los últimos años sobre el exilio remitimos al artículo de Jordi Font Agulló y Jordi Gaitx Moltó, «L'exili de 1939. Un estat de la qüestió entre dues conmemoracions (2009-2014)», FranquismeぬTransiciò, 2, 2014, pp. 231-280. Y para el caso de los historiadores Edoardo Tortarolo, «Historians in the Storm. Emigré Historiography in the Twentieth Century», Matthias Middell, Lluís Roura (eds.), Transnational Challenges to National History Writing, Palgrave Macmillan, 2013, pp. 377-403. 
De todos modos, y como ya se ha anunciado al comienzo del texto, la ocupación de Francia por las tropas alemanas nazis posibilitó que prosperase una denuncia contra él que supuso su encarcelamiento. El Cónsul español en Burdeos, Enrique Beltrán Manrique, se dirigía el 10 de noviembre de 1939 al decano de la Faculté des Lettres de Bordeaux, para recordarle que dos solicitantes españoles optaban a la plaza de Lector de español: Amada López de Meneses y Manuel Núñez de Arenas. Le informaba de que este último había participado en la Junta encargada de la depuración de la Enseñanza bajo el Ministerio del comunista Jesús Hernández, «de sinistre mémoire». También alegaba «que cet espagnol ennemi de l'Espagne» no tiene ninguna relación con el consulado, al contrario que la candidata, «bonne espagnole» y con una admirable carrera ${ }^{33}$.

Once días después llegó la respuesta del decano, quien argumentó al cónsul: cuando G. Delpy lo propone ${ }^{34}$, no conoce otra candidatura más que la de Núnez de Arenas, ya que López de Meneses no había presentado ninguna solicitud. Además, tiene los títulos necesarios, ha realizado diferentes estancias en Francia, ha seguido varios cursos en esa Facultad, y está casado con una francesa. No solo eso: sobre su actitud en Francia, están convencidos de que es «correcte et discrète». Defiende así la elección de Manuel Núñez de Arenas como Lector de Español, aunque reconoce que debido a las circunstancias excepcionales del momento, no se ha seguido el cauce habitual de selección de lectores a través del Office des Universités Françaises, situación que espera se reanude pronto.

Pese a que en la siguiente carta del 27 de noviembre de 1939 Enrique Beltrán agradece las explicaciones del decano sobre el nombramiento del «sujet espagnol Nuñez de Arenas", insiste en que Amada López de Meneses presentó en julio de 1939 una petición como lectora de español en el Office des Universités Françaises, citando varias ciudades de Francia, entre ellas Burdeos.

El asunto queda así, y la última carta de este año ( 5 de diciembre) es la dirigida de nuevo por el Cónsul al Embajador de España en París, remitiéndole copia de toda esta correspondencia sobre «uno de los asesores del comunista Jesús Hernández desde el Ministerio de Instrucción Pública de la zona española marxista».

Pero la segunda remesa de correspondencia que comienza el 2 de febrero de 1943 se produce en un escenario muy diferente, y va a acarrear consecuencias directas para Núñez de Arenas. En esta ocasión, la delación y los ajustes de cuentas profesionales encontraron resonancia en el clima político de persecución de «rouges espagnols» en Burdeos. El madrileño ya había sido retenido por las autoridades alemanas durante algunas semanas, pero fue puesto en libertad gracias a "gestiones oficiales por parte de ciertas

33. AGA, Sección Asuntos Exteriores, 54/1139. Para todas las citas hasta el final del artículo. El nombre de la aspirante no debió de resultar del todo desconocido para el cuerpo de hispanistas de Burdeos, ya que habían aparecido varios artículos suyos en el Bulletin Hispanique, donde seguirá colaborando (publicó en 1935, 1938, 1942, 1946 y 1951).

34. Gaspard Delpy, antiguo miembro de l'EHEH, había sucedido a Georges Cirot como titular de la cátedra de español en Burdeos. 
Autoridades españolas en Francia», una liberación que sorprendió en el propio consulado. La policía alemana volvió entonces a detenerlo al conocer los datos del Gobierno español. Y tras los informes pertinentes entre el Ministerio de Asuntos Exteriores en Madrid, la Embajada de España en Vichy y el Consulado de España en Burdeos, se procedió a solicitar oficialmente a las autoridades francesas su destitución el 17 de marzo de 1943 como Lector de Español. En este intercambio de informaciones, se describe así el perfil del historiador desde la Dirección de Relaciones Culturales del Ministerio al embajador Félix de Lequerica (2-2-1943):

[...] Se trata de un periodista rojo, [...] conocido como elemento comunista [...]. Aunque en apariencia lleva una vida retirada y no se tienen noticias de que se aproveche su cargo para hacer propaganda de sus ideas, en la sombra trabaja activamente en sentido comunista y en el café exterioriza sus opiniones en tal sentido.

Consiguió el cargo que desempeña, valiéndose de amistades destacadas en la política local de Burdeos y en competición con otros españoles apoyados por nuestra Representación Oficial en aquella Ciudad.

Y al respecto escribe el agente de policía español agregado al Consulado de Burdeos, que tiene «la casi seguridad de que este hombre es peligroso por sus ideas y por su actuación, tratándose de persona inteligente y cultivada. De ello me he de servir para lograr nuestros propósitos».

M. Núńez de Arenas fue recluido en 1942 y 1943 en las cárceles de La Santé y Fresnes. Su plaza de Lector la ocupó en un primer momento M. Prieto, lector en el Lycée de Bordeaux. Poco después lo hizo un candidato oficial con "garantías» pactado con las autoridades españolas, Ángel Baselga Yarza, quien no se reincorporó tras pasar las fiestas de Navidad de 1944 en Espańa. Mientras tanto, Núnez de Arenas a punto estuvo de ser "canjeado» por un desertor alemán detenido en el campo de concentración de Miranda de Ebro, una iniciativa alemana que no tuvo éxito ${ }^{35}$.

\section{De Burdeos a París: Le Professeur NúÑez de Arenas}

Reincorporado finalmente a la Facultad de Burdeos tras su liberación en 1944, Georges Cirot lo saludaba así desde la «Chronique» del Bulletin Hispanique:

35. Según la documentación se trataba de Dahm Horst Jurgen, natural de Dusseldorf, y detenido en el campo de concentración de Miranda de Ebro, bajo el nombre de Duval. En Carta del Cónsul de España en Burdeos al Embajador de España en Vichy (Burdeos, 15 octubre 1943), AGA, Sección Asuntos Exteriores, 54/1139. Matilde Eiroa y Concha Pallarés, «Uncertain fates: allied soldiers at the Miranda de Ebro Concentration Camp", The Historian, Vol. 76, Issue 1, pp. 26-49; Matilde Eiroa, «Refugiados extranjeros en España: el campo de concentración de Miranda de Ebro", Ayer, no 57, 2005, pp. 125-152. 
[...] emprisonné trois fois par la Gestapo, privé de son poste par ordre supérieur, il a été réintégré en novembre dernier. Ancien élève du Lycée de Bordeaux, connaissant le français comme sa propre langue, très épris de recherches et très consciencieux, il est, comme personne, en mesure d'initier à la pratique de l'espagnol nos étudiants, auxquels depuis huit ans le séjour en Espagne est devenu de plus en plus impossible. Nous tenons à dire les services quil a rendus et qu'il rendra encore dans un enseignement qui se développe et qui prend décidément, dans le secondaire comme dans le supérieur, l'importance justifiée par tant de raisons culturelles, politiques et économiques ${ }^{36}$.

Allí permaneció hasta 1948, año en el que se trasladó a París como investigador del Centre National de la Recherche Scientifique y profesor de l'École Normale Supérieure de Saint-Cloud, continuando la relación a través de sus artículos en el Bulletin Hispanique.

En aquella época coincidió en la capital francesa con los historiadores Emilio Gómez Nadal y su esposa Teresa Andrés, Manuel Tunón de Lara y F. G. Bruguera, todos ellos miembros de la Unión de Intelectuales Españoles, colaboradores habituales en su Boletín y miembros de su comité de redacción. Fue un periodo donde podían recordar en las tertulias semanales de los animados cafés del Boulevard Saint-Michel la ya lejana guerra civil durante la que coincidieron en Valencia, comentar sus experiencias en los campos de concentración españoles y franceses, $y$, ante todo, discutir sobre su papel como historiadores en la lucha antifranquista o la articulación de sus investigaciones históricas en un país ajeno. De hecho, el juego de transferencias entre la historiografía liberal y obrera de principios de siglo en España, la influencia del hispanismo y la historiografía francesa así como su recepción y adaptación entre los jóvenes historiadores españoles de los años 70 se encuentra en este escenario del exilio.

Será durante el entierro de Núñez de Arenas en París, en 1951, cuando se produzca el primer contacto entre Pierre Vilar y Manuel Tunón de Lara, iniciándose entre ellos una fructífera relación personal y profesional. Y en 1953, dos años después de su repentina muerte, Francisco G. Bruguera publicó su Histoire de l'Espagne. Incomprensiblemente aún sin traducir hoy en día, contiene las claves de la historia económica y social de España en las que incidirá Manuel Tunón de Lara en sus Historias de España de los siglos XIX y XX (1961, 1966). Así, en esta temprana fecha, Bruguera se adelantaba en la genealogía de una historia del movimiento obrero realizada en Francia por exiliados españoles y que servirá como modelo en el interior. Y se adelanta también Bruguera en su dedicatoria al reunir en ella a los futuros referentes en la historiografía mundial y española respectivamente: Fernand Braudel y Pierre Vilar; y al brindársela "À la mémoire du professeur Manuel Nuñez de Arenas, mon vieil ami, mort en exil». Comenzaba aquí la fortuna de la figura y la obra de Manuel Núñez de Arenas.

36. Georges Cirot, «Chronique», Bulletin Hispanique, XLVI, juillet-décembre 1944, n. 2, p. 293. 
